\title{
Acute abdominal pain in emergency room: Is it always a simple diagnosis?
}

\author{
Bahjat Barakat, ${ }^{1}$ Francesco Bacci, ${ }^{2}$ Raffaele Pezzilli ${ }^{3}$ \\ ${ }^{1}$ Emergency Department; ${ }^{2}$ Department of Oncology and Hematology; ${ }^{3}$ Department of Gastroenterology, \\ Sant'Orsola-Malpighi Hospital, Bologna, Italy
}

\begin{abstract}
Acute abdominal pain is characterized by pain arising from the abdominal area, of non-traumatic origin with a maximum duration of five days and represents a true medical emergency. It is also one of the most common reasons for referral to an emergency department and the most common cause for no trauma-related hospital admissions. Hematologic disorders represent the $0.3 \%$ of all admissions for acute abdominal pain. We report a rare case of retroperitoneal bulky mass due to anaplastic large T-cell lymphoma. This entity represents a rare tumor and early diagnosis leads to a correct diagnosis of the origin of the acute abdominal pain and chemotherapy is vital to ensure good prognosis.
\end{abstract}

\section{Introduction}

Acute abdominal pain is characterized by pain arising from the abdominal area, of non-traumatic origin, with a maximum duration of five days and represents a true medical emergency. ${ }^{1}$ It is also one of the most common reasons for referral to an emergency department and the most common cause for no trauma-related hospital admissions. ${ }^{2,3}$

In addition, abdominal pain represents the chief complaint at entry for about $8 \%$ of the total visits in the emergency department in the United States ${ }^{4}$ and for about $9 \%$ of the cases in Italy. ${ }^{5}$ In

Correspondence: Raffaele Pezzilli, Department of Gastroenterology, Sant'Orsola-Malpighi Hospital, Via Massarenti 9, 40138 Bologna, Italy. E-mail: raffaele.pezzilli@aosp.bo.it

Key words: Large T cell lymphoma; Abdominal pain; Retroperitoneum; Non-Hodgkin lymphoma.

Contributions: the authors contributed equally.

Conflict of interest: the authors declare no potential conflict of interest.

Funding: none.

Received for publication: 11 May 2018.

Revision received: 28 August 2018.

Accepted for publication: 30 August 2018.

This work is licensed under a Creative Commons Attribution 4.0 License (by-nc 4.0).

(C) Copyright B. Barakat et al., 2018

Licensee PAGEPress, Italy

Emergency Care Journal 2018; 14:7552

doi:10.4081/ecj.2018.7552
Italy, its prevalence is significantly higher in females than in males as well as in foreign over Italian patients. Even if the most frequent emergency department diagnoses is non-specific abdominal pain, other type of pain especially that associated with abdominal mass may be encountered. ${ }^{5}$ Thus, we believe of interest report this case representative of difficulties in the emergency room to evaluate the abdominal pain.

\section{Case Report}

An 18-year-old man, coming from Ivory Coast, presented with a long standing (about one year) acute recurrent episodes of diffuse abdominal pain increasing after eating and associated with significant weight loss $(8 \mathrm{~kg})$. He had no fever, complained constipation and joint pain of the wrists. Him past medical history was positive for spontaneous right pneumothorax and previous epigastric weapon wound. Physical examination showed acute epigastric pain without splenomegaly of presence of lymphadenopathy. Biochemical blood test such as white blood cell count, blood urea nitrogen, creatinine, albumin, total bilirubin, transaminases, and lactate dehydrogenase value were within the normal limits. Abdominal ultrasonography (US) showed the presence of a heterogeneous solid mass localized in the in mesogastrium of $13 \mathrm{~cm}$ in size with a central blood vessel (Figures 1 and 2); no US alterations of liver, spleen, pancreas and kidneys were found. For better evaluation of the abdominal mass, the patient underwent contrast enhanced computed tomography (CT) scan that confirmed the presence and the size of the retroperitoneal mass (Figure 3A) without vascular infiltration and showing a compression of the jejunum (Figure 3B). Chest X-ray was normal as well as both the electrocardiogram and echocardiography.

A CT-positron emission tomography was also carried out showing a clear intense hyperaccumulation of the radiotracer in the abdominal mass (SUV max 20.8) and in the Glisson's capsule (SUV max 17.5) and in retroperitoneal lymph nodes (SUV max 5.9) (Figure 4).

An ultrasound-guided fine-needle aspiration of the abdominal mass was performed (Figure 5). Predominant population of large cells with irregular nuclei, some hallmark cells with eccentric kidney shaped nuclei. All malignant cells are strongly positive for CD30 (T phenotype) and anaplastic lymphoma kinase negative. Bone marrow biopsy revealed a normal picture. Patient was also negative for HIV antibodies, Quantiferon test, cytomegalovirus, Epstein and Barr virus, $\mathrm{B}$ and $\mathrm{C}$ hepatitis viruses, and strongyloides; serum tumor markers (Ca 19-9 and CEA) had also normal levels.

The final diagnosis of the retroperitoneal bulky mass was anaplastic large cell lymphoma in stage IV (with involvement of lymph-nodes and liver) B (with systemic symptoms) ${ }^{6}$ and a CHOEP chemotherapy (Cyclophosphamide $750 \mathrm{mg} / \mathrm{m} 2 \mathrm{IV}$ on day 1 Doxorubicin $50 \mathrm{mg} / \mathrm{m} 2 \mathrm{IV}$ on day 1 Vincristine (Oncovin) $2 \mathrm{mg}$ 
IV on day 1, Etoposide $100 \mathrm{mg} / \mathrm{m} 2$ IV on days days 1-3 Prednisone $100 \mathrm{mg}$ per os on days 1 to 5) was carried out. After 12 months the patient is still alive and in good general health.

\section{Discussion and Conclusions}

Hematologic disorders represents the $0.3 \%$ of all admissions for acute abdominal pain. ${ }^{5}$ In addition the case we have reported, i.e. retroperitoneal bulky mass was anaplastic large cell lymphoma

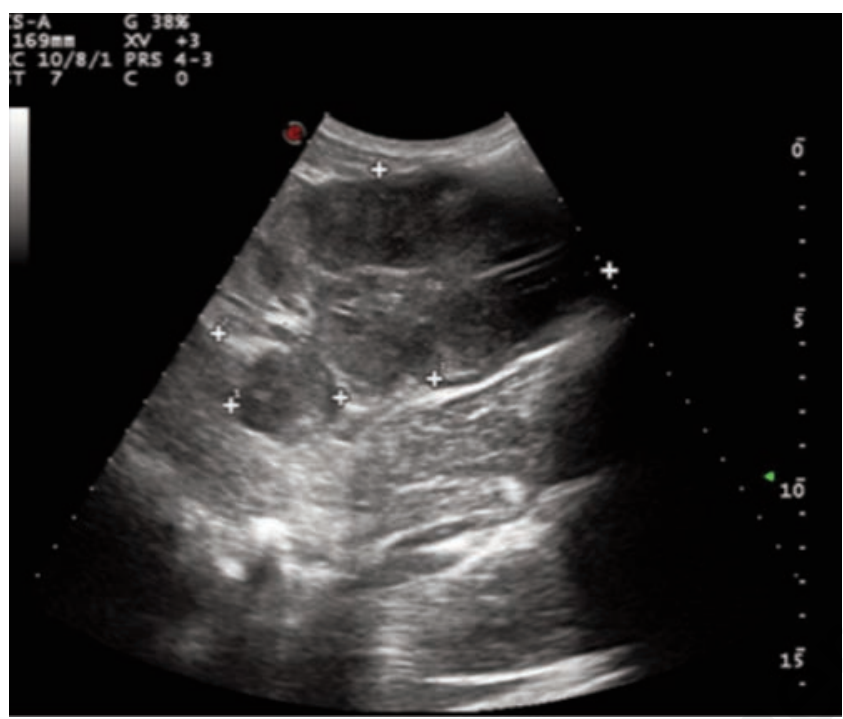

Figure 1. Ultrasonography showing the large abdominal mass delimited with + . represents a rare tumor ${ }^{7}$ being the larger series reported by Pileri et $a l .{ }^{8}$ Some clinical characteristics of this case are superimposable to those previously reported: 5 foreign young male patient, without Italian medical assistance, with high severe abdominal pain, having more diagnostic imaging procedure to reach a diagnosis.

According to the International Agency for Research on Cancer, the age standardized incidence rate of non-Hodgkin lymphoma between both sexes worldwide is estimated at 5.0 per 100,000 people. The differential incidence between more and less developed regions of the world is more pronounced than that of mortality rate (2.3 and 2.7 per 100,000 respectively) ${ }^{9}$

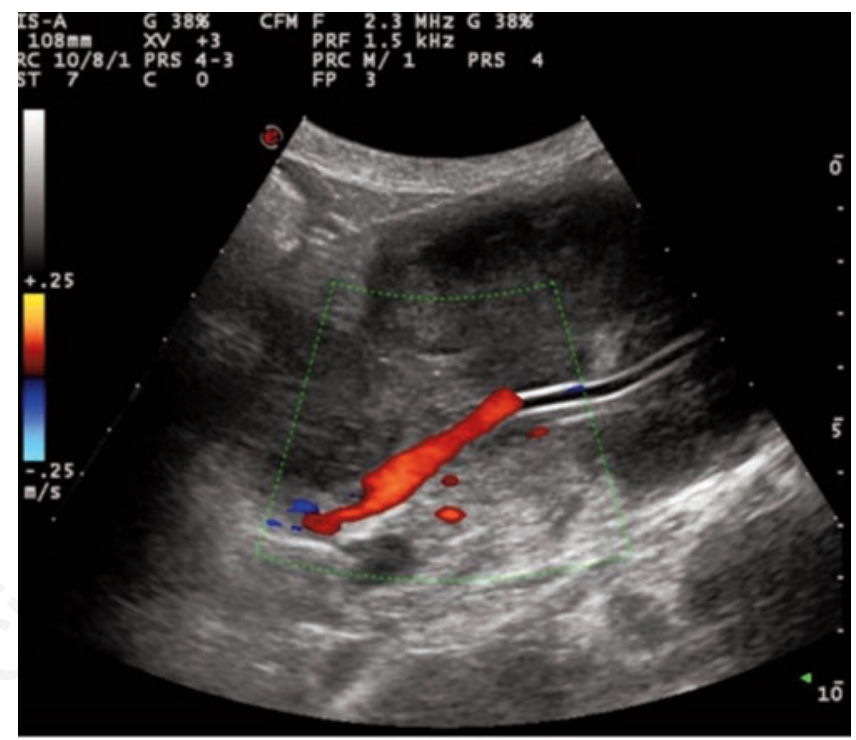

Figure 2. Echo-color-Doppler of the abdominal mass showing a central blood vessel.

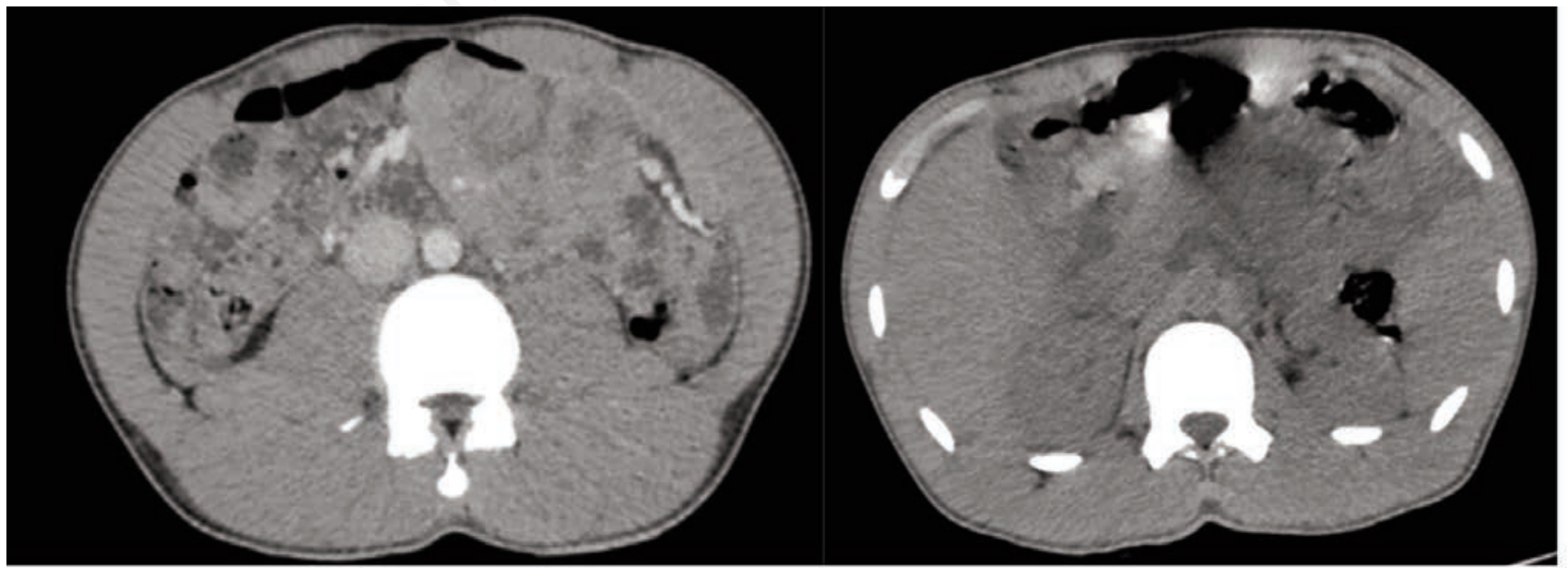

A

B

Figure 3. Contrast enhanced computed tomography showing (A) the abdominal mass without vascular infiltration and showing a compression of the jejunum (B). 


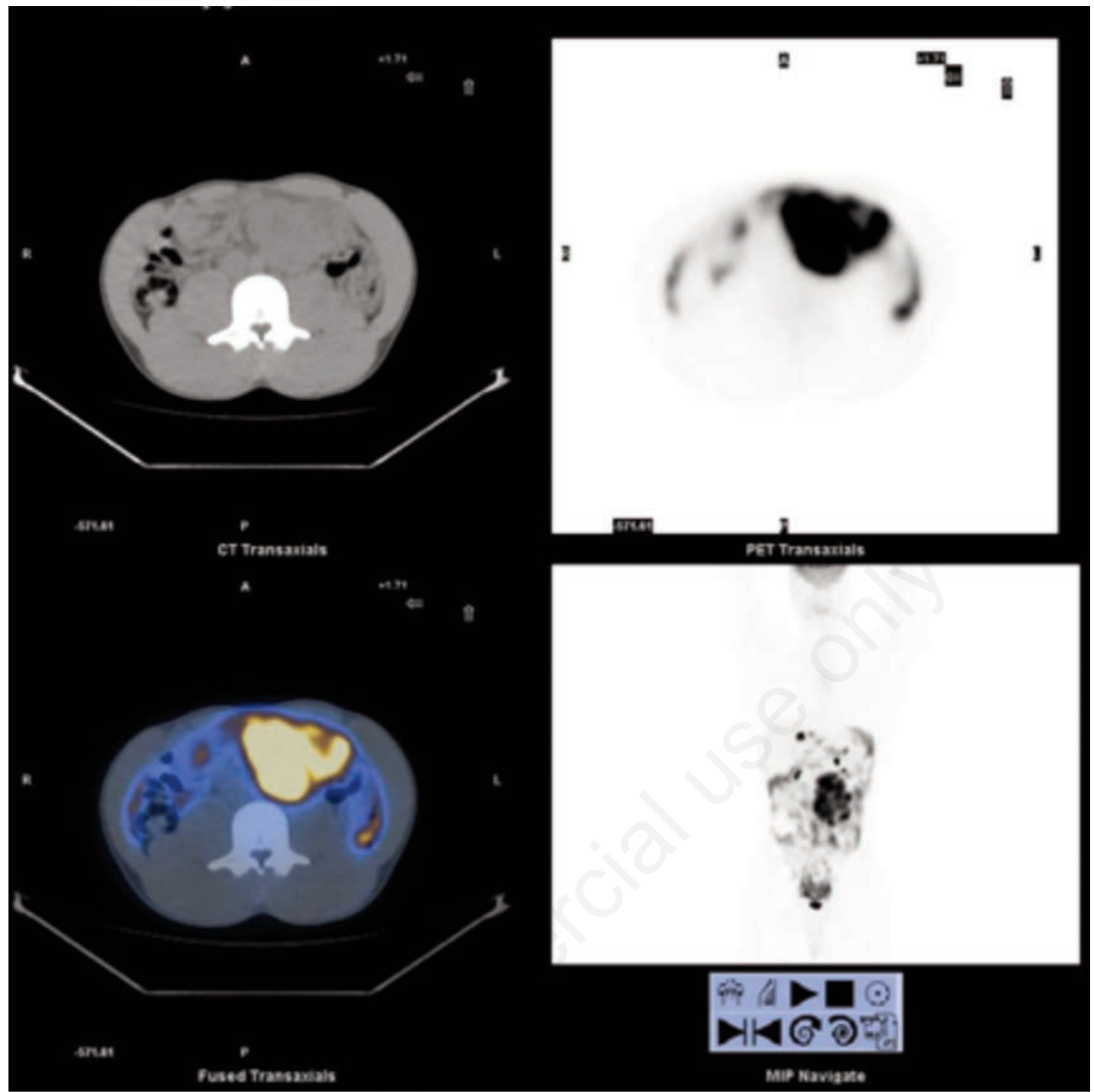

Figure 4. Computed tomography-positron emission tomography showing a clear intense hyperaccumulation of the radiotracer in the abdominal mass (SUV max 20.8) and in the Glisson's capsule (SUV max 17.5) and in retroperitoneal lymph nodes (SUV max 5.9).

Patients with Stage IV Ann Arbor disease constituted a total of $49.3 \%$. The median overall survival is 47 months and the median event free survival is 32 months. ${ }^{10,11}$

The large primary retroperitoneal T-cell lymphoma we have described is a rare tumor and the gastrointestinal symptoms may be better evaluated, especially in emergency due to acute abdominal pain. In conclusion, we should be aware that early diagnosis leads to a correct diagnosis of the origin of the acute abdominal pain and chemotherapy is vital to ensure good prognosis as in our case classified as Stage IV-B.

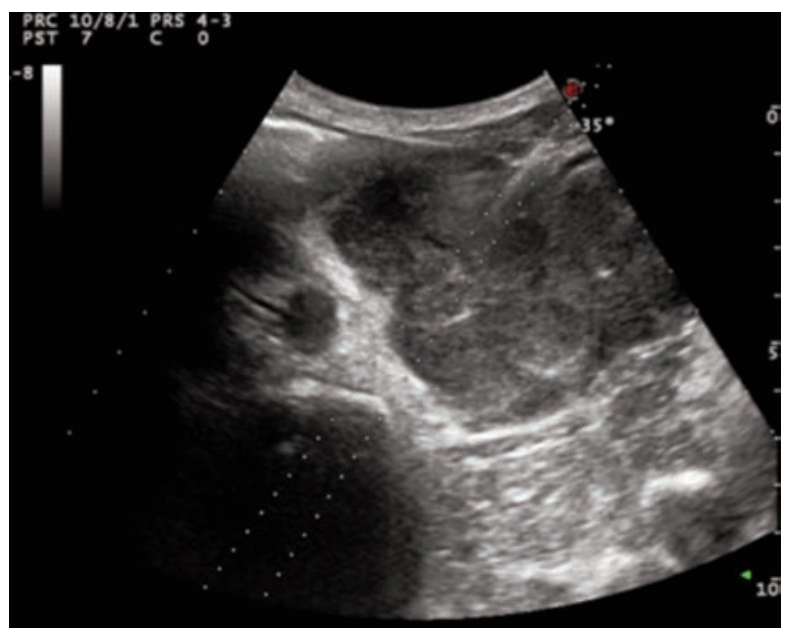

Figure 5. Ultrasound-guided fine-needle aspiration of the abdominal mass. 


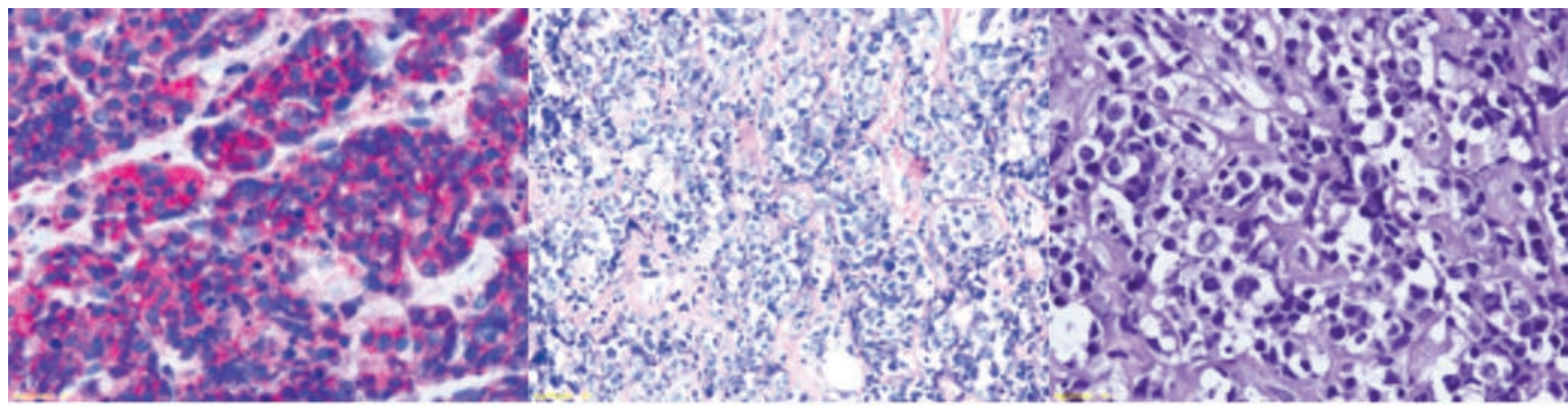

A

B

C

Figure 6. (A, B) Predominant population of large cells with irregular nuclei, some hallmark cells with eccentric kidney shaped nuclei (hematoxylin eosin stain, Giemsa stain); (C) all malignant cells are strongly positive for CD30 (T phenotype).

\section{References}

1. Gans SL, Pols MA, Stoker J, et al. Guideline for the diagnostic pathway in patients with acute abdominal pain. Dig Surg 2015;32:23-31.

2. Trentzsch H, Werner J, Jauch KW. Acute abdominal pain in the emergency department a clinical algorithm for adult patients. Zentralbl Chir 2011;136:118-28.

3. Macaluso CR, McNamara RM. Evaluation and management of acute abdominal pain in the emergency department. Int J Gen Med 2012;5:789-97.

4. National Center for Health Statistics (NCHS). National Hospital Ambulatory Medical Care Survey: 2010 Emergency Department Summary Table. Centers for Disease Control and Prevention. Available from: http://www.cdc.gov/nchs/data/ ahcd/nhamcs_emergency/2010_ed_web_tables.pdf Accessed 15 May 2015.

5. Caporale N, Morselli-Labate AM, Nardi E, et al. Acute abdominal pain in the emergency department of a university hospital in Italy. Unit Eur Gastroenterol J 2016;4:297-304.
6. Edge SB, Byrd DR, Compton CC, et al. AJCC Cancer Staging Manual. 7th ed. New York, NY: Springer; 2010.

7. Ravindhran B, Prakash C, Govindharaj S, et al. An aggressive primary retroperitoneal diffuse large b-cell lymphoma mimicking a pancreatic neoplasm, presenting as duodenal stenosis. J Clin Diagn Res 2017;11:PD09-11.

8. Pileri SA, Zinzani PL, Ascani S, et al. Diffuse large B-cell lymphoma with primary retroperitoneal presentation: clinicopathologic study of nine cases. Ann Oncol 2001;12:1445-53.

9. Ferlay J, Soerjomataram I, Dikshit R, et al. Cancer incidence and mortality worldwide: sources, methods and major patterns in GLOBOCAN 2012. Int J Canc 2015;136:E359-86.

10. Nimmagadda RBV, Digumarti R, Nair $R$, et al. Histopathological pattern of lymphomas and clinical presentation and outcomes of diffuse large B cell lymphoma: A multicenter registry based study from India. Indian J Med Paediatr Oncol 2013;34:299-304.

11. Khera R, Jain S, Kumar L, et al. Diffuse large B-cell lymphoma: experience from a tertiary care center in north India. Med Oncol 2010;27:310-8. 